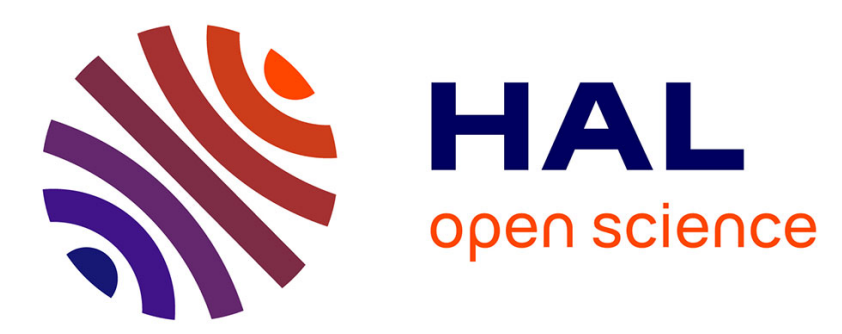

\title{
Tuning the adhesive geometry of neurons: length and polarity control.
}

Caterina Tomba, Celine Braini, Beilun Wu, Nir Gov, Catherine Villard

\section{To cite this version:}

Caterina Tomba, Celine Braini, Beilun Wu, Nir Gov, Catherine Villard. Tuning the adhesive geometry of neurons: length and polarity control.. Soft Matter, 2014, 10 (14), pp.2381. 10.1039/c3sm52342j . hal-00987841

\section{HAL Id: hal-00987841 https://hal.science/hal-00987841}

Submitted on 6 May 2014

HAL is a multi-disciplinary open access archive for the deposit and dissemination of scientific research documents, whether they are published or not. The documents may come from teaching and research institutions in France or abroad, or from public or private research centers.
L'archive ouverte pluridisciplinaire HAL, est destinée au dépôt et à la diffusion de documents scientifiques de niveau recherche, publiés ou non, émanant des établissements d'enseignement et de recherche français ou étrangers, des laboratoires publics ou privés. 


\title{
Tuning the adhesive geometry of neurons : length and polarity control $^{\dagger}$
}

\author{
Caterina Tomba, ${ }^{a, b}$ Céline Braïni, ${ }^{a}$ Beilun Wu, ${ }^{a}$ Nir S. Gov, ${ }^{* c}$ and Catherine Villard ${ }^{* a}$
}

\author{
Received Xth XXXXXXXXXX 20XX, Accepted Xth XXXXXXXXX 20XX \\ First published on the web $X t h X X X X X X X X X X 200 X$ \\ DOI: 10.1039/b000000x
}

Neurons acquire their functional and morphological axo-dendritic polarity by extending, from competing minor processes (neurites), one long axon among numerous dendrites. We employed complementary sets of micropatterns built from 2 and $6 \mu \mathrm{m}$ wide stripes of various length to constrain hippocampal neuron shapes. Using these geometries, we have (i) limited the number of neuronal extensions to obtain a minimal in vitro system of bipolar neurons, and (ii) controlled neurite width during growth by the generation of a progressive cell shape asymmetry on either side of the cellular body. From this geometrical approach, we gained a high level of control of each neurite length and of the localization of axonal specification. To analyze these results, we developed a model based on a width and polarization dependent neurite elongation rate and on the existence of a critical neurite length that sets the axonal fate. Our data on the four series of micro-patterns developed for this study are described by a single set of growth parameters, well supported by experiments. The control of neuronal shapes by adhesive micro-patterns thereby offers a novel paradigm to follow the dynamical process of neurite lengthening and competition through the process of axonal polarization.

\section{Introduction}

Neurons are branched cells whose various stages of growth can be monitored in vitro. A fundamental step of neuronal development is the acquisition of polarity which results in both a morphological and functional distinction between numerous dendrites and one long axon. In cultured hippocampal neurons, primary undifferentiated neuronal branches, or neurites, compete for axonal fate by a race for the highest elongation rate $^{1}$. It is now well established that the various aspects of neuronal growth including branching, growth rate and axonal specification can be modulated by physical cues. Of note, these cues can be provided by the architecture of the brain itself, with the striking example of the in vivo migrating neurons constrained to adopt a bipolar geometry in their journey along micron-wide elongated glial cells ${ }^{2}$. While the mechanical rigidity has proven to tune branching when approaching the extreme softness of the brain tissue ${ }^{3}$, topography provided by micro-pillars can increase the neurite elongation rate and thereby bias neuritic competition ${ }^{4}$. Geometries imposed by

$\dagger$ Electronic Supplementary Information (ESI) available: [details of any supplementary information available should be included here]. See DOI: 10.1039/b000000x/

${ }^{a}$ Univ. Grenoble Alpes, Inst NEEL, F-38042 Grenoble, France. CNRS, Inst NEEL and CRETA, F-38042 Grenoble, France.

$b$ Laboratoire des Technologies de la Microelectronique, CNRS-Universit Joseph Fourier, CEA-LETI, Grenoble, France.

$c$ Department of Chemical Physics, Weizmann Institute of Science, Rehovot, Israel; E-mail: nir.gov@weizmann.ac.il. adhesive micro-patterns are also an efficient way to control neuronal growth and to impose the localization of axonal specification, either by constraining neuronal shapes ${ }^{5}$ or by superimposing to these shapes a chemical gradient of adhesion ${ }^{6}$. Poly-lysine constitutes a widely used polycationic coating on glass ${ }^{7}$ although laminin, a component of the extra-cellular matrix, is also commonly employed to foster axonal growth ${ }^{8}$. Interestingly, varying the neurite width from 2 to $4 \mu \mathrm{m}$ seems to have some influence on both neuronal growth and polarization ${ }^{6}$. However a simple picture of the role of this geometric parameter would deserve an extensive study.

The nascent axon is not only the longest neurite, it also presents molecular specificities according to a characteristic spatial organization. In particular, a structure named the axonal initial segment (AIS) plays the role of a diffusion barrier at the axon base to ensure the establishment and the maintenance of polarity ${ }^{9}$. The characteristic length of the AIS (30$40 \mu \mathrm{m})$ is also found in axotomy experiments where the remaining stump retains a memory of its axonal nature only when longer than $35 \mu \mathrm{m}^{10}$. A length threshold of $40 \mu \mathrm{m}$ seems also to be required to stabilize the polarized state ${ }^{11,12}$.

In this work, we performed an in-depth study of the role of the neurite width in the process of neurite elongation and axonal specification. In that aim, we used a geometric variations on the theme of a generic bipolar geometry imposed by polyL-lysine micro-patterns. This non-specific coating against dendrite or axonal elongation was specifically chosen to dispose of a system where the shape is the only control parameter. 
Four series of micro-patterns allowing changes in the surface of adhesion between and along competing neurites were designed. The lengths of neurites were systematically measured, and the possible axonal nature of these neuronal extensions were assessed using the usual Tau-immunostaining ${ }^{13} 14$. We provide for the first time experimental data that allow to follow the dynamical neurite lengthening and competition through the process of axonal polarization. These data were compared to a simple model based on a width-dependent elongation rate modulated by a distance over which a neurite has the highest probability to become an axon. By unraveling the relation between neuronal shapes and growth, our results support the existence of a critical length, close to the AIS length, that controls the axonal fate of a neuronal extension. Most of all, we provide in this work an original experimental paradigm to probe the impact of geometry on polarization.

\section{Adhesive geometries and cells}

We designed a minimal system of neurons characterized by two diametrically opposite neurites. This bipolar cell geometry was obtained by growing in vitro hippocampal neurons (see the Experimental section) on Poly-L-lysine (PLL) adhesive stripes intercalated with $14 \mu \mathrm{m}$ PLL disks designed to anchor the cell body (or soma). All patterns derived from an initial geometry built from $2 \mu \mathrm{m}$ and $6 \mu \mathrm{m}$ wide stripes located on each side of the central disk (6:2 pattern). Then, the length of either the $2 \mu \mathrm{m}$ or the $6 \mu \mathrm{m}$ wide stripe is progressively reduced and replaced by a $6 \mu \mathrm{m}$ or $2 \mu \mathrm{m}$ wide portion of length $l$, respectively. Patterns differ from the localization of these

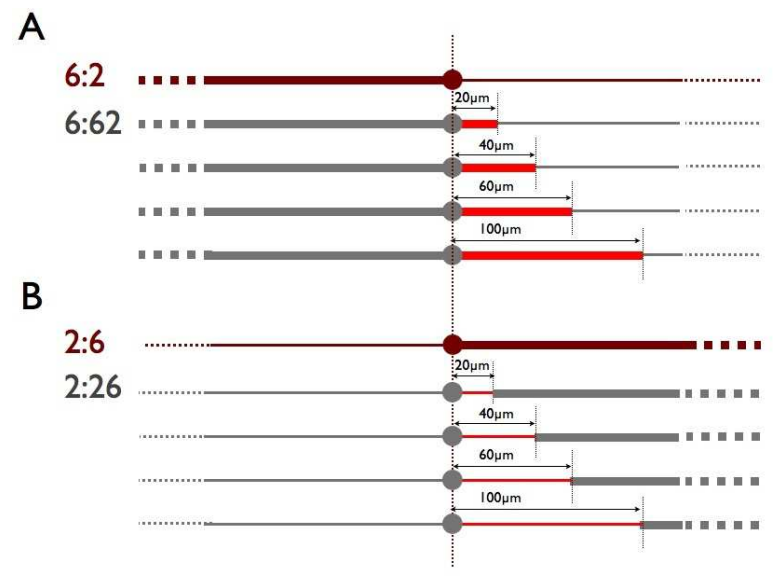

Fig. 1 Progressive evolution of the 6:2 (2:6) pattern (dark red) from a geometrical modification initiated from the central disk. A) The 6:2 pattern (top) and the derived 6:62 patterns formed by the addition of a $6 \mu \mathrm{m}$ wide portions of various lengths (in red). B) The 2:6 pattern (top) and the derived 2:26 patterns formed by the addition of a $2 \mu \mathrm{m}$ wide portions of various lengths (in red). portions and are divided into two categories. In the first set of patterns (Fig.1), the $6 \mu \mathrm{m}$ (Fig. 1a) or $2 \mu \mathrm{m}$ (Fig. 1b) wide portions of length $l$ depart from the circular disk (i.e. from the soma). In the second set of patterns (Fig. 2), the non-modified portions of the $6: 2$ pattern on the contrary remains near the adhesive disk. All patterns are defined by a simple nomenclature of type $x: x y$ or $y$ :xy expressing the spatial succession of the 2 and $6 \mu \mathrm{m}$ wide stripes.

As an example, the typical morphology of cells grown on

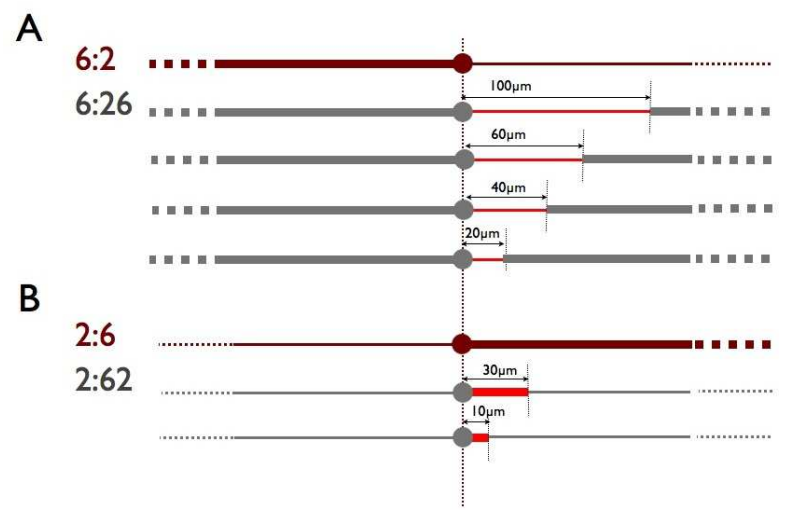

Fig. 2 Progressive evolution of the 6:2 (2:6) pattern (dark red) from a geometrical modification initiated away from the central disk. A) The 6:2 pattern (top) and the derived 6:26 patterns. In red, the remaining $2 \mu \mathrm{m}$ wide stumps of various lengths from the central disk. B) The 2:6 pattern (top) and the derived 2:62 patterns. In red, the remaining $6 \mu \mathrm{m}$ wide stumps of various lengths from the central disk.

the 2:26 patterns are given on Figure 3. In this particular set of neurons, only the longest neuron (top) grown on the geometry characterized by $l=20 \mu m$ expresses the characteristic axonal Tau gradient on its longest, $2 \mu m$ wide neurite. Other examples of Tau staining are displayed in Fig. S1.

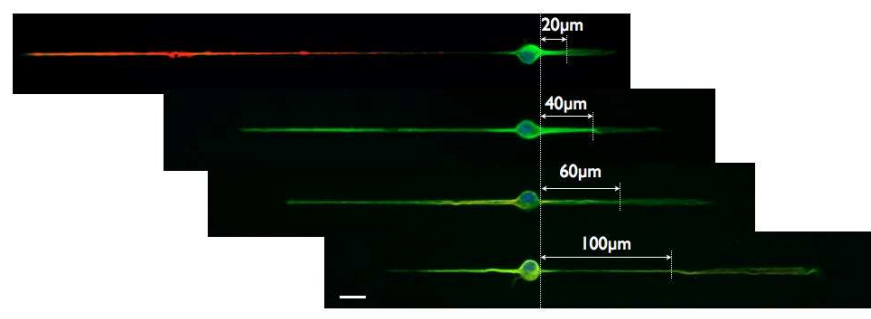

Fig. 3 Four neurons illustrating the four different geometries (from top to bottom, $l=20,40,60$ and $100 \mu \mathrm{m}$, respectively) associated to the 2:26 pattern. The position and length of the various $2 \mu \mathrm{m}$ wide stumps emerging from the central disk are indicated on each image. Microtubules (YL1/2, green), nucleus (Hoechts, blue) and axon (Tau, red). Scale bar : $20 \mu \mathrm{m}$. 


\section{Theoretical Model}

We model the elongation of the neurites along the adhesive strips, by considering the following observations:

1. The growth velocity is affected by the width of the adhesive stripe on which the neurite is growing. We will consider the simplest scenario where the growth material synthetized at the soma level has to be distributed along the available surface at the neurite growth-cone, leading to a larger (smaller) velocity $v_{t i p}$ when the width is smaller (wider).

2. The neurites have a certain probability to turn into axons, which signals for the whole cell to polarize. This polarization event seems to depend on the absolute length of the neurite ${ }^{11,12}$.

3. Following the polarization event the neurite that was "chosen" to become the axon may continue to grow at a different growth velocity $\beta v_{\text {tip }}$ (with $\beta \geq 1$ ). The other neurite(s) continues to grow at a reduced growth velocity $\gamma v_{\text {tip }}(0 \leq \gamma \leq 1)$, following the polarization event.

We now turn these assumptions into a calculation of the mean neurite length on the patterns shown in Figs.1,2. For this we need to assume some probability density function that describes the probability for the neurite to undergo polarization, as a function of its length. Based on the notion of a critical length for this transition ${ }^{11,12}$, we consider the following simple step-like cumulative probability to polarize

$$
\begin{aligned}
P_{p o l}(x) & =\frac{1}{2}\left(1+\tanh \left[\frac{x-L_{p o l}}{\sigma_{p o l}}\right]\right) \\
\Rightarrow p_{p o l}(x) & =\frac{d P_{p o l}(x)}{d x}=\frac{1}{2 \sigma_{p o l}} \operatorname{sech}\left[\frac{x-L_{p o l}}{\sigma_{p o l}}\right]^{2}
\end{aligned}
$$

where $L_{p o l}$ is the critical polarization length and $\sigma_{p o l}$ gives the variance of the probability distribution function around this critical length, as shown in Fig.4.

We then divide the calculation into two cases, for the case of the right neurite polarizing, and for the left. We now demonstrate the calculations that result from our model for the $\mathrm{x}$ :xy patterns (shown in Fig.1). A similar calculation follows along the same lines for the x:yx patterns. For polarization along the right neurite, the length of the right neurite is given by the following

$$
L_{R, R}= \begin{cases}x_{p o l}+\left(T-t_{p o l, 1}\right) \beta v_{y}, & {\left[x_{p o l}>l\right]} \\ l+\left(T-t_{l}\right) \beta v_{y}, & {\left[x_{p o l}<l, T>t_{l}\right]} \\ l+\left(T-t_{p o l, 2}\right) \beta v_{x}, & {\left[x_{p o l}<l, T<t_{l}\right]}\end{cases}
$$

where $l$ is the length of the $x$-segment on the right side, $x_{p o l}$ is the location of the polarization event on the right neurite, $T$ is

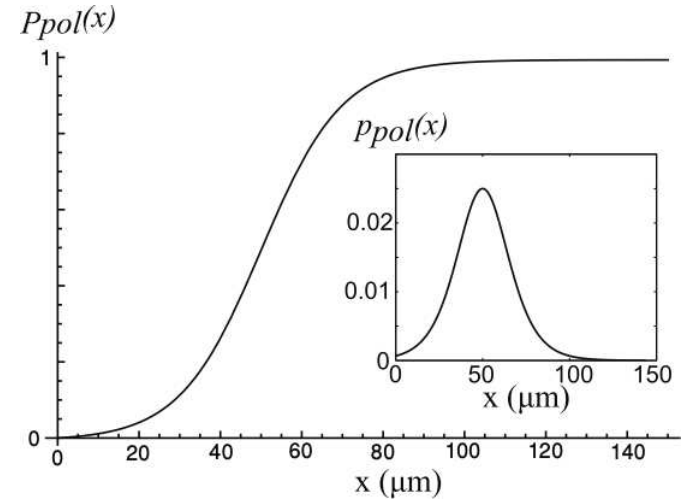

Fig. 4 Polarization cumulative probability $P_{p o l}(x)$ (Eq.1), and the probability distribution function $p_{p o l}(x)$ (Eq.2) (inset).The parameters used are: $L_{p o l}=50 \mu \mathrm{m}, \sigma_{p o l}=20 \mu \mathrm{m}$.

the time at which the observation is performed and $v_{x, y}$ are the tip velocities on the respective width. The different times are defined as

$$
\begin{aligned}
t_{p o l, 1} & =\frac{l}{v_{x}}+\frac{x_{p o l}-l}{v_{y}} \\
t_{p o l, 2} & =\frac{x_{p o l}}{v_{x}} \\
t_{l} & =t_{p o l, 2}+\frac{l-x_{p o l}}{\beta v_{x}}
\end{aligned}
$$

The maximal value of $x_{p o l}$ for the right neurite is

$$
x_{p o l, \max , R}= \begin{cases}l+\left(T-\left(l / v_{x}\right)\right) v_{y}, & {\left[T>\left(l / v_{x}\right)\right]} \\ T v_{x}, & {\left[T<\left(l / v_{x}\right)\right]}\end{cases}
$$

Over the possible range of $x_{p o l}$ we need to integrate the lengths in Eq. 3 multiplied by the probability that the right neurite polarizes at position $x_{p o l}$, which is given by $p_{p o l}(x)$ (Eq.2). In addition we need to multiply by the probability that the competing neurite has not already polarized itself by this time. This probability is given by the cumulative probability up to the position of the tip on the left neurite $x_{p o l, L}$ corresponding to $x_{p o l}$ on the right neurite

$$
\begin{aligned}
p_{\text {nopol }, L}\left(x_{p o l}\right) & =1-\int_{0}^{x_{p o l, L}} p_{p o l}(x) d x \\
& =1-\frac{1}{2}\left(\tanh \left[\frac{L_{p o l}}{\sigma_{p o l}}\right]-\tanh \left[\frac{L_{p o l}-t_{p o l} v_{x}}{\sigma_{p o l}}\right]\right)
\end{aligned}
$$

where $t_{p o l}$ stands for $t_{p o l, 1}, t_{p o l, 2}$ given in Eqs.4,5 respectively, and $x_{p o l, L}=t_{p o l} v_{x}$.

The final mean length that we get is

$$
\left\langle L_{R, R}\right\rangle=\int_{0}^{x_{p o l, m a x}, R} L_{R, R} p_{p o l}\left(x_{p o l}\right) p_{\text {nopol }, L}\left(x_{p o l}\right) d x_{p o l}
$$


where the overall probability for polarization of the right neurite is given by

$$
P_{R}=\int_{0}^{x_{p o l, m a x, R}} p_{p o l}\left(x_{p o l}\right) p_{n o p o l, L}\left(x_{p o l}\right) d x_{p o l}
$$

For completeness, the overall probability for polarization of the left neurite is given by

$$
P_{L}=\int_{0}^{x_{p o l, \max , L}} p_{p o l}\left(x_{p o l}\right) p_{\text {nopol }, R}\left(x_{p o l}\right) d x_{p o l}
$$

where: $x_{p o l, \max , L}=T v_{x}$, and

$$
p_{\text {nopol }, R}\left(x_{p o l}\right)=1-\int_{0}^{x_{p o l, R}} p_{p o l}(x) d x
$$

$=$

$$
\begin{cases}1-\frac{1}{2}\left(\tanh \left[\frac{L_{p o l}}{\sigma_{p o l}}\right]-\tanh \left[\frac{L_{p o l}-x_{p o l}}{\sigma_{p o l}}\right]\right), & {\left[l>x_{p o l}\right]} \\ 1-\frac{1}{2}\left(\tanh \left[\frac{L_{p o l}}{\sigma_{p o l}}\right]-\tanh \left[\frac{L_{p o l}-\left(l+\left(x_{p o l}-l\right) * v_{y} / v_{x}\right)}{\sigma_{p o l}}\right]\right), & {\left[l<x_{p o l}\right]}\end{cases}
$$

In a similar manner we calculate the length of the left neurite when the right one is polarized $\left(\left\langle L_{L, R}\right\rangle\right)$ and the lengths for the case of polarization on the left neurite (i.e. $\left\langle L_{L, L}\right\rangle,\left\langle L_{R, L}\right\rangle$ ). In order to get the total mean length along each side, we also need to consider the lengths of the neurites when no side has polarized. For the $x: x y$ these lengths are given by

$$
L_{L, 0}=T v_{x} P_{\text {nopol }}
$$

and

$$
L_{R, 0}= \begin{cases}\left(l+\left(T-\left(l / v_{x}\right)\right) v_{y}\right) P_{\text {nopol }}, & {\left[T>\left(l / v_{x}\right)\right]} \\ T v_{x} P_{\text {nopol }}, & {\left[T<\left(l / v_{x}\right)\right]}\end{cases}
$$

where the probability for no polarization is given by: $P_{\text {nopol }}=$ $1-P_{R}-P_{L}$, where $P_{R}$ is given in Eq. 11 .

Summing the lengths on each neurite from all the possible polarization outcomes gives the final mean lengths: $L_{L}=$ $L_{L, 0}+\left\langle L_{L, R}\right\rangle+\left\langle L_{L, L}\right\rangle$ and $L_{R}=L_{R, 0}+\left\langle L_{R, R}\right\rangle+\left\langle L_{R, L}\right\rangle$.

\section{Results}

The model presented above requires a set of parameters, namely the culture duration $(T)$, the elongation rates of neurites constrained by either 2 and $6 \mu \mathrm{m}$ wide stripes, the multiplicative coefficients controlling the change of these elongation rates after axonal specification ( $\beta$ for the axon, $\gamma$ for the dendrite), and the characteristic lengths of the probability distribution function $L_{p o l}$ and $\sigma_{p o l}$. The three first parameters are fixed by the experiments, with (i) $T=72 \mathrm{hrs}$ (all neurons were fixed and analyzed after 3 days of culture), and (ii) $v_{6}=2 \mu \mathrm{m} / \mathrm{hr}$ and $v_{2}=4 \mu \mathrm{m} / \mathrm{hr}$, as roughly indicated from the data presented in Fig5 showing the evolution of the total neurite length for cells grown on symmetric $x: x$ patterns $(\mathrm{x}=2,4$ and $6 \mu \mathrm{m})$.
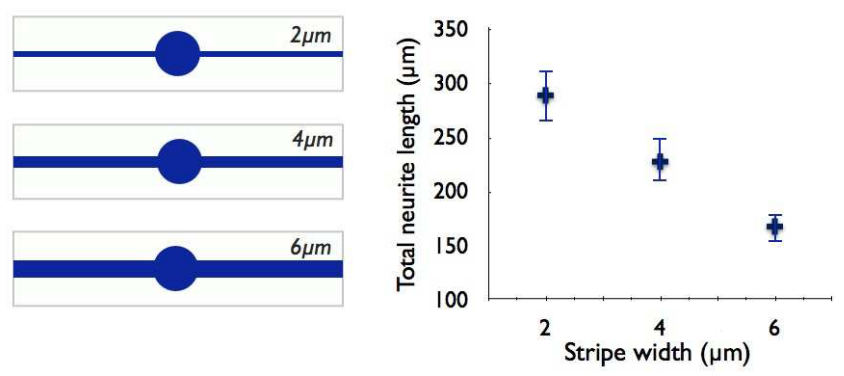

Fig. 5 Influence of the stripe width on the total neurite length after 3 days of culture. The geometries used are sketched beside the graph. Errors bars denote the standard deviation. .

Fitting our model to the experimental data while keeping within the available constraints on the values of the different parameters, we were able to obtain the values of $L_{p o l}, \sigma_{p o l}, \gamma$ and $\beta$.

We want to emphasize here the limiting case of the 2:6 pattern. From the analysis of 180 neurons coming from 3 different cultures performed in the same conditions, 161 exhibited a Tau immunostaining on the $2 \mu \mathrm{m}$ wide stripe. Strikingly, none displayed this marker on the $6 \mu \mathrm{m}$ wide side (of note, the $6 \mu \mathrm{m}$ wide neurite is always the shortest) (see for example Fig.6B, red symbols). Therefore, the 2:6 pattern offers for the first time and to the best of our knowledge the first demonstration that the localization of axonal specification can be reliably set by geometrical constraints on the neurite width. Of note, neurons plated on the four different $x: x y$ or $y: x y$ sets of patterns were grown under slightly different conditions of culture (different serum batch, mice type, temperature set-point of the incubator) that prevents a comparison of the length data obtained from these neurons with the ones of the 2:6 pattern. Nevertheless, we made the choice to show on the same graphs the polarization data coming from all patterns, including the 2:6 geometry .

In Fig.6A we compare the calculations of length presented in the previous section with the experimental data for the $6: 62$ pattern. In Fig.6B we compare the calculated polarization probabilities $P_{L}, P_{R}$ to the observed frequency of axons identified. In Figs.7,9,8 we compare the calculated neurite lengths and the polarization probabilities to the observations for the other three patterns shown in Figs.1,2. The points corresponding to the 2:6 pattern in Figs. 8 and 9 are arbitrary located at 
the coordinate $l=200 \mu \mathrm{m}$ (and naturally at $l=0 \mu \mathrm{m}$ in Figs.6 and 7).

For all the cases we used the same set of parameters, given in the caption of Fig. 6 .
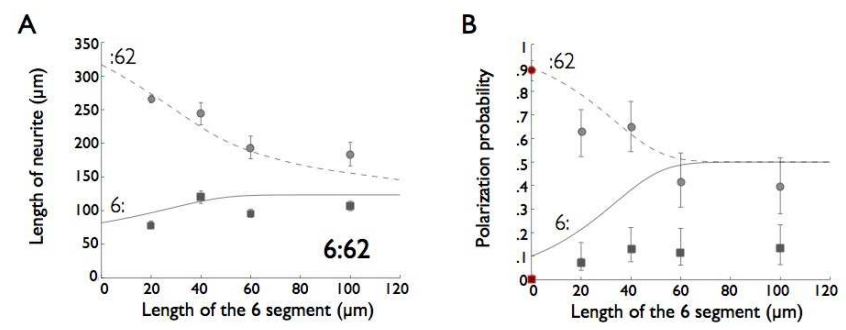

Fig. 6 Patterns 6:62. A) Neurite lengths. B) Polarization probabilities. All results after three days of growth. Symbols denote the experimental data and lines the result of the model calculation: Circles (dashed line, $L_{R}$ ) along the right neurite (here the :xy branch, ) and squares (solid line, $L_{L}$ ) along the left neurite (here the $\mathrm{x}$ : branch). The red symbols correspond to the 2:6 pattern. The parameters used in this calculation are: $T=72 \mathrm{hrs}, v_{6}=2 \mu \mathrm{m} / \mathrm{hr}$, $v_{2}=4 \mu \mathrm{m} / \mathrm{hr}, \beta=1.2, \gamma=0.4, L_{p o l}=50 \mu \mathrm{m}, \sigma_{p o l}=20 \mu \mathrm{m}$.
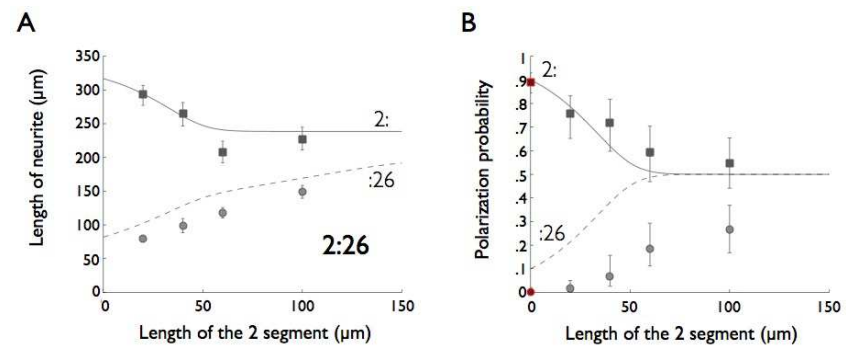

Fig. 7 Patterns 2:26. A) Neurite lengths. B) Polarization probabilities. All results after three days of growth. Symbols denote the experimental data and lines the result of the model calculation: Circles (dashed line, $L_{R}$ ) along the right neurite (here the :xy branch,) and squares (solid line, $L_{L}$ ) along the left neurite (here the $\mathrm{x}$ : branch). The red symbols correspond to the 2:6 pattern. The parameters used in this calculation are the same as in Fig.6.

\section{Discussion}

We first emphasize that the lengths corresponding to the four categories of patterns, namely 6:62, 2:26, 6:26 and 2:62, were fitted by the same set of parameters. Among them, only $L_{p o l}$, $\sigma_{p o l}, \beta$ and $\gamma$ were free when running our model.

The fits displayed in Figs.6,7,9,8 give for the two first parameters the values $L_{p o l}=50 \mu \mathrm{m}$ and $\sigma_{p o l}=20 \mu \mathrm{m}$. The critical polarization length $L_{p o l}$ is therefore similar to the value of the AIS (i.e. $30-40 \mu \mathrm{m}$ ) found experimentally in ${ }^{11,12}$, although a little bit higher. Note that the probability to polarize
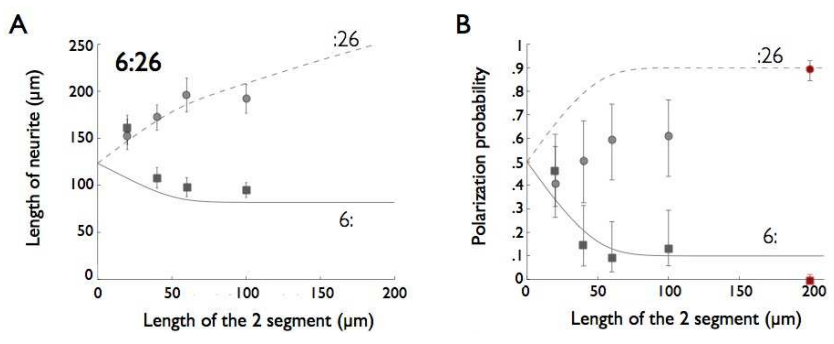

Fig. 8 Pattern 6:26. A) Neurite lengths. B) Polarization probabilities. All results after three days of growth. Symbols denote the experimental data and lines the result of the model calculation: Circles (dashed line, $L_{R}$ ) along the right neurite (here the :xy branch,) and squares (solid line, $L_{L}$ ) along the left neurite (here the $y$ : branch). The red symbols correspond to the 2:6 pattern. The parameters used in this calculation are the same as in Fig.6.
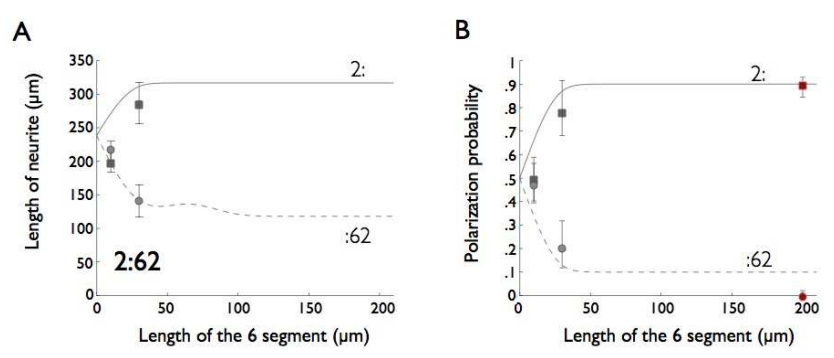

Fig. 9 Patterns 2:62. A) Neurite lengths. B) Polarization probabilities. All results after three days of growth. Symbols denote the experimental data and lines the result of the model calculation: Circles (dashed line, $L_{R}$ ) along the right neurite (here the :xy branch,) and squares (solid line, $L_{L}$ ) along the left neurite (here the $y$ : branch). The red symbols correspond to the $2: 6$ pattern. The parameters used in this calculation are the same as in Fig.6.

into an axon has a significant spread around this critical length (see Fig.4), in coherence with the experimental data reported in ${ }^{15}$. Interestingly, it has been shown that a differential neurite elongation obtained using adhesive geometries where only one neurite among four is allowed to extend beyond a length of $\geq 60 \mu \mathrm{m}$ is highly favorable for the axonal specification of the longest neurite ${ }^{16}$. Our findings are in very good agreement with these recents results that also indicate the existence of a critical length for the establishment of the neuronal axodendritic polarity.

Then, some specific modalities of hippocampal neuron growth first described by Ruthel and Banker ${ }^{17} 18$ and more recently by Flynn et al. ${ }^{19}$ might provide a better understanding of the significance of the parameters $\beta$ and $\gamma$. These works report that neurite elongation is mediated by periodic growth spurts produced by propagative growth-cone like structures (so-called waves) when reaching the neurite tip. We indeed observed the presence of waves in neurons on micro-patterns 
(see Fig. S2 and the associated movie in the Supplementary Materials). Interestingly, these authors found a difference in the periodicity of waves and in the amplitude of the waveassociated growth spurts between the nascent axon and the other minor processes (see Fig. 2 in ref. ${ }^{19}$ ). From these specificities of waves, we expect a growth ratio between the nascent axon and the other neurites of about $\sim 2-4$, which is in agreement with the value $\beta / \gamma=3$ that came out of our model. We therefore emphasize that the adjustable parameters used in the model show a biological relevance.

We explored the sensitivity of our model to its adjustable parameters in the particular example of the 6:62 patterns (see Fig. S3 and commentaries). One important feature here is that the polarization probability is very sensitive to $L_{p o l}$. Interestingly, higher lengths (and higher polarization probabilities on the :62 branch) can be obtained for the shortest $6 \mu \mathrm{m}$ wide stumps when increasing $L_{p o l}$. This result is a direct consequence of the fact that, for relatively large $L_{p o l}$ values, the polarization decision is taken when the future axon tip has already reached the $2 \mu \mathrm{m}$ wide portion. This increases the :62 branch probability to experience the fastest possible elongation rate $\beta \times v_{t i p_{2}}$ during the period $T$ of growth.

The length data are well fitted by our model. The trend of the polarization rate is also correctly described by the model, although a systematic shift can be observed (see for example results on the 6:26 pattern, Fig.8B). To discuss the polarization fits, we first emphasize that the identification of polarized neurons, i.e. of axons, is based on the observation of specific markers, in our case Tau ${ }^{13}{ }^{14}$. This microtubule binding protein is distributed along a gradient revealing the distal part of the axon, about $100 \mu \mathrm{m}$ away and more from the soma (see Fig.3). Interestingly, the largest discrepancies between the model and the experimental data are observed when the Tau-staining should appear on a $6 \mu \mathrm{m}$ wide portion. In this condition of unusual neurite spreading (the spontaneous neurite diameter is indeed $\sim 1-2 \mu \mathrm{m}$ on uniformly adhesive surfaces), the establishment of a Tau gradient might be impaired. To test this hypothesis, we additionally counted as axons the longest neurite in neurons that present a strong length asymmetry (i.e. a ratio greater than 2 between the neurite lengths) without however showing a Tau-staining. With these new set of data we significantly reduced the shifts (see Fig. S4 and the particular improvement obtained for the 6:26 pattern) without however obtaining a perfect match between the model and the experimental data. This point therefore remains an open issue.

\section{Experimental}

\subsection{Neuron culture, labelling and imaging}

Embryonic day E(18) hippocampi were dissected and then dissociated in MEM medium supplemented with $10 \%$ horse serum, 1\% L-Glutamine, $1 \%$ Sodium pyruvate and $0.05 \%$ Peni-streptomycine (Invitrogen). Neurons were plated on Poly-L-Lysine (Sigma, P2636) coated coverslips and kept first (for 3 hours) in the dissociation medium and then in the maintenance medium of Neurobasal supplemented with $2 \%$ B27, $1 \%$ L-Glutamine and $0.05 \%$ Peni-streptomycine (Invitrogen). Neurons were fixed in $4 \%$ paraformaldehyde and immunostained with standard techniques, after a permeabilization step of 30 minutes in PBS supplemented with 2\% BSA and $0.25 \%$ Triton. The following antibodies and respective secondary antibodies were used in the indicated dilutions: for microtubules, rat anti-tubulin antibody (clone YL1/2, 1:500) and Alexa488 coupled (Invitrogen, 1:250), for axons, Tau (clone Tau-1, Millipore, 1:500) and CY3 coupled (Invitrogen, 1:250), for DNA, Hoechst (Invitrogen, 1:1000).

Isolated fixed neurons were analyzed with a right Olympus BX51 microscope.

\subsection{Patterning}

Poly-L-lysine patterns were transferred on silanized substrates $\left(18 \times 18 \mathrm{~mm}^{2}\right.$ coverslips from VWR, ref. 631-1331). After an oxygen plasma cleaning step (50W) of $2 \mathrm{~min}$ in duration, silanization was performed in a liquid phase using the 3Methacryloxypropyltrimethoxysilane (Bind-Silane, Sigma). Then, patterns were defined using UV classical photolithography steps, including Shipley S1805 photoresist spinning (4000 rpm, $0.5 \mu \mathrm{m}$ thickness, $115^{\circ} \mathrm{C}$ annealing step for $1 \mathrm{~min}$ ), insulation through a mask, development (Microposit concentrate 1:1, Shipley), Poly-L-lysine deposition ( $1 \mathrm{mg} / \mathrm{ml}$ over night), and lift-off using an ultra-sound ethanol bath.

\section{Conclusions}

By combining experimental and modeling approaches of neuron growth and polarization under constrained geometries, this work provide an original system to explore some fundamental aspects of neuronal biology. In particular, using the proposed model we conclude that the tip elongation rate in neurites is width dependent. This finding should direct future research to reveal the underlying mechanism. One possible direction could be to explore the width-dependence of the propagative growth cone like structures (waves) that are associated to neurite elongation in these neurons ${ }^{17}$. Finally, although indications for the existence of a critical length for axonal polarization already exist ${ }^{16}$, our work indicates that this length, unlike 
the tip velocity, does not depend on the neurite width. This gives some new insights into the still mysterious phenomena of the establishment of axo-dendritic polarity. As a whole, by combining a novel experimental set-up and a simple physical model, our work helps to identify several key properties of neurite growth and polarization, that a more microscopic model would have to account for. Furthermore, we have shown that by controlling the neurite width we can achieve an unequaled control over the localization of the axonal branch. This finding could have practical implications for the manipulation of in vitro neurons and the design of controlled neuronal micro-circuits.

\section{Acknowledgements}

This work was funded by ANR-07-PCVI-0007 and the Nanosciences Fundation (Grenoble). We acknowledge support from the NanoFab facility of the Institut Néel and CNRS funding for the sabbatical stay of Prof. Nir S. Gov at the Institut Néel. We are indebted to A. Triller and his group, especially S. Colasse, for discussions and support in neuron culture.

\section{References}

1 Dotti CG, Sullivan CA, Banker GA . The establishment of polarity by hippocampal neurons in culture. Neuroci. 1988, 8, 1454.

2 Neuronal migration in the adult brain: are we there yet? Troy Ghashghaei H, Lai C, Anton ES. Nat. Rev. Neurosci. 2007 8:141-151

3 Flanagan LA, Ju YE, Marg B, Osterfield M, Janmey PA. Neurite branching on deformable substrates. Neuroreport. 200213 (18):2411-5.

4 Micholt L, Grtner A, Prodanov D, Braeken D, Dotti CG, et al. Substrate Topography Determines Neuronal Polarization and Growth In Vitro. PLOS ONE 2013 8(6): e66170. doi:10.1371/journal.pone.0066170.

5 S. Roth, M. Bisbal, J. Brocard, G. Bugnicourt, A. Andrieux, S. GoryFauré S, and C. Villard. How morphological constraints affect axonal polarity in mouse neurons. PLoS One 2012, 7:e33623.

6 Fricke R, Zentis PD, Rajappa LT, Hofmann B, Banzet M, Offenhusser A, Meffert SH. Axon guidance of rat cortical neurons by microcontact printed gradients Biomaterials 2011 32:2070-6.

7 Ruegg UT,Hefti F. Growth of dissociated neurons in culture dishes coated with synthetic polymeric amines. Neurosci Lett 1984 49:319-24.

8 Esch T, Lemmon V, and Banker G. Local presentation of substrate molecules directs axon specification by cultured hippocampal neurons. J Neurosci 1999 19(15) 6417-26.

9 C. Leterrier et al., End-binding proteins EB3 and EB1 link microtubules to ankyrin G in the axon initial segment Proc. Natl. Acad. Sci., 2011, 108, 8826-31

10 S. Gomis-Rüth, C. J. Wierenga, and F. Bradke, Plasticity of Polarization: Changing Dendrites into Axons in Neurons Integrated in Neuronal Circuits, Curr Biol, 18, 992-1000.

11 D. Seetapun and D.J. Odde, Cell-length-dependent microtubule accumulation during polarization. Curr Biol 2010, 20, 979-88.

12 H. Yamamoto, T. Demura, M. Morita, G.A. Banker, T. Tanii, and S. Nakamura. Differential neurite outgrowth is required for axon specification by cultured hippocampal neurons. J Neurochem. 2012, 123, 904-10.

13 K. S. Kosik and E. A. Finch, MAP2 and Tau Segregate into Dendritic and Axonal Domains After the Elaboration of Morphologically Distinct
Neurites: An lmmunocytochemical Study of Cultured Rat Cerebrum $J$ Neurobiol 1987, 7, 3142-3153

14 Black MM, Slaughter T, Moshiach S, Obrocka M, Fischer I. Tau is enriched on dynamic microtubules in the distal region of growing axons. $J$ Neurosci 1996 16:3601-3619.

15 Wainrib G, Thieullen M, and Pakdaman K. Intrinsic variability of latency to first-spike. Biol Cybern 2010 103:43-56.

16 Yamamoto $\mathrm{H}$ et al., Differential neurite outgrowth is required for axon specification by cultured hippocampal neurons J Neurochem. 2013. 123(6): 904-910

17 G. Ruthel and G. Banker, Actin-dependent anterograde movement of growth-cone-like structures along growing hippocampal axons: a novel form of axonal transport? Cell Motil Cytoskel. 1998, 40, 160-173.

18 Ruthel G., G. Banker. Role of moving growth cone-like "wave" structures in the outgrowth of cultured hippocampal axons and dendrites. J Neurobiol 1999, 39, 1-153.

19 K. C. Flynn, C. W. Pak, A. E. Shaw, F. Bradke, and J. R. Bamburg, Growth cone-like waves transport actin and promote axonogenesis and neurite branching. Developmental Neurobiology 2009, 69, 761-779. 